\title{
A Multivariate Analysis-Factors Related To Vascular Calcification On Regular Hemodialysis Patient; First In Indonesia
}

Daniel Reinaldo P. Situmorang ( $\sim$ dr.situmorang@yahoo.co.uk)

Divisi Nefrologi dan Hipertensi Departemen Ilmu Penyakit Dalam Universitas Sumatera Utara https://orcid.org/0000-0001-5165-0917

Riri Andri Muzasti

Universitas Sumatera Utara

Syafrizal Nasution

Universitas Sumatera Utara

Netty Delvrita Lubis

Universitas Sumatera Utara

Research article

Keywords: Chronic Kidney Disease, Hemodialysis, Vascular Calcification, Fetuin-A

Posted Date: September 3rd, 2019

DOI: https://doi.org/10.21203/rs.2.11874/v1

License: (9) This work is licensed under a Creative Commons Attribution 4.0 International License. Read Full License 


\section{Abstract}

Introduction: Cardiovascular disease is one of the main causes of death in patients with chronic kidney disease (CKD) undergoing hemodialysis or peritoneal dialysis therapy. This is related to calcification in large blood vessels such as the aorta. Although some known coronary risk factors, such as age, dyslipidemia, diabetes and smoking, play an important role in cardiovascular disease in patients undergoing hemodialysis, but several other risk factorsy such as anemia, uremic toxins, oxidative stress, and disorders of mineral and bone metabolism also associated with CKD.Method: This study was an analytical cross-sectional study with subjects were from CKD patients who underwent regular hemodialysis (for at least 3 months) in hemodialysis unit of RSKG Rasyida Medan from May 2018 and aged $>18$ years. Interviews were conducted regarding age, duration of hemodialysis. Laboratory examination of calcium, phosphate and fetuin-A levels and lateral abdominal X-ray examination were conducted, then a statistical analysis was performed to determine the relationship of these factors with blood vessel calcification in regular hemodialysis patients.Results: Of 113 subjects, duration of hemodialysis in no calcifications group was $52.09 \pm 21.725$, while mild grade calcification underwent HD for $51.81 \pm 29.115$, and severe calcification group underwent HD for $69.06 \pm 36.030$. A total of 10 patients without calcification had a history of DM, which were total 14 patients with calcification (mild and severe group) had history of DM. The mean serum calcium level in the group without calcification was $9,467 \pm$ $0,843 \mathrm{mg} / \mathrm{dl}, 9,638 \pm 0,708 \mathrm{mg} / \mathrm{dl}$ in mild group, while the average calcium level was 5,412 $\pm 0,645 \mathrm{mg}$ / dl in severe group.Conclusion: Duration of hemodialysis, diabetes mellitus, calcium levels and fetuin-A significantly influence the occurrence of calcification in the abdominal blood vessels. $(p=0.004, p=$ $0.049, p=0.005$ and $p=0.004$ )

\section{Background}

Cardiovascular disease is one of the main causes of death in patients with chronic kidney disease (CKD) undergoing hemodialysis or peritoneal dialysis therapy. Increased mortality caused by cardiovascular disease, associated with calcification in large blood vessels, such as the aorta, where this can cause stiffness in the arteries and increase pulsation pressure and decreased myocardial perfusion at diastole. ${ }^{1}$

Therefore, prevention of cardiovascular disease is important to reduce the incidence of morbidity and mortality. Although some known coronary risk factors, such as age, dyslipidemia, diabetes and smoking, play an important role in cardiovascular disease in patients undergoing hemodialysis, but several other risk factors, which are associated with chronic kidney disease are also involved, such as anemia, uremic toxins, oxidative stress, and mineral and bone metabolic disorders. ${ }^{2}$ For this reason, many researchers are now finding out the relationship between a disorder of mineral metabolism and bone and vascular calcification.

The mechanism of ectopic calcification is very diverse and is not clearly known. The most studied is the pathology of vascular calcification as a major risk factor for cardiovascular mortality. Basically, 
regulation of calcification is regulated by maintaining extracellular calcium and phosphate concentration. ${ }^{3}$

There are many methods that can be use to diagnose a vascular calcification such as ultrasound mostly for superficial vascular, multi-slice computed tomography (MSCT) but the downside is it cannot differentiate a intima or media tunica calcification, and lateral lumbal X-ray. There are no any gold standard to diagnose a vascular calcification. Although it is still a controversy, The Kidney Disease Improving Global Outcome (KDIGO) suggest a lateral abdominal radiography can be use for detection of vascular calcification. ${ }^{4}$

\section{Methods}

\section{Study Samples}

Samples were from populations of CKD patients who underwent regular hemodialysis in the hemodialysis unit of RSKG Rasyida Medan from May 2018 (for at least 3 months) and aged> 18 years. The subject had received information and gave consent to participate in informed and voluntary research. Patients who underwent irregular hemodialysis or were unstable were excluded from this study.

\section{Study Design}

This study is an analytical cross-sectional study. The proportion formula is used to determine the number of samples. After obtaining approval from the ethics committee, the subjects who met the inclusion and exclusion criteria were given an explanation and asked to provide informed consent to take part in the study. Interviews were then conducted to determine the age, history of diabetes mellitus and the duration of the patient's hemodialysis. Then blood sampling is performed to determine serum calcium levels and serum phosphorus, as well as lateral abdomen x-ray. After the data collected, data processing and data analysis were then conducted.

\section{Statistical Analysis}

Univariate analysis was conducted to obtain an overview of each variable studied. Bivariate analysis is used to state the analysis of two variables, which are the dependent variable and the independent variable. To see the strength of the relationship between the dependent and independent variables, the value of the Prevalence Ratio (RP) is used. In bivariate analysis anova test is used because the independent variable is numerical data and the dependent variable is ordinal data.

Multivariate analysis is used to see the relationship of several independent variables with the dependent variable by connecting independent variables with the dependent variable at the same time. In this analysis, the variables with the greatest influence can be known, the form of relationships between variables, related directly or indirectly to other variables. 


\section{Results}

\section{Research Subjects Characteristic Distribution}

This study was followed by 113 patients as research subjects who had met the inclusion and exclusion criteria. The majority of the study subjects were men (59.3\%) with a median age of 57 years. Based on the results of laboratory tests, the median serum calcium level was $9.8 \mathrm{mg} / \mathrm{dl}$, the mean serum phosphate level was $5.39 \mathrm{mg} / \mathrm{dl}$. From the results of lateral abdominal X-ray examination in this study the majority of patients having severe calcification of $43.4 \%$, no calcifications of $38.1 \%$ ), and only $18,6 \%$ having mild calcification. (Table 1)

\section{Relationship between Research Subject Characteristics with the Degree of Blood Vessel Calcification}

Man having more severe calcification (47.8\%), compared with women who showed the same results in severe calcification and no calcification (each of $37.0 \%$ ). Based on the statistical test, it was concluded that there was no significant difference between gender and the degree of calcification $(p=0.211)$. (Table 2)

Subjects with no calcification were $49.0 \pm 13.020$ years of age, lower than the average age of mild and severe degrees $(54.81 \pm 9.19$ and $59.47 \pm 8.615)$. Based on the statistical test, it was concluded that there were significant differences between mean of age to the degree of calcification ( $p=0.001)$ (Table 2$)$. In Table 3 there was a significant difference in age at normal levels compared to severe degrees $(p=0.001)$.

The subjects who have no calcifications had underwent hemodialysis for $52.09 \pm 21.725$, the average HD duration of mild calcification degree was $51.81 \pm 29.115$, while the average length of HD degrees of severe calcification was $69.06 \pm 36.030$. It was concluded that there was a difference in the average duration of HD to the degree of calcification $(p=0.004)$. In table 3 there is a significant difference between the no calcification and severe calcification $(p=0.007)$ and between mild calcification and severe calcification $(p=0.007)$.

10 subjects with no calcification had a history of DM more than of mild and severe group (8 and 6 people) so it was concluded that there was a correlation between the history of DM and the degree of calcification $(p=0.049)$.

Subjects who did not have calcifications had an average serum calcium level of 9,467 $\pm 0,843 \mathrm{mg} / \mathrm{dl}$, the average level of mild calcium was $9,638 \pm 0.708 \mathrm{mg} / \mathrm{dl}$, while the average level of calcium in severe calcification group was $5.412 \pm 0.645 \mathrm{mg} / \mathrm{dl}$. It can be concluded that there are significant differences between the average calcium level and the degree of calcification $(p=0.005)$. In table 3 there is a significant difference between the degree of absence of calcification and severe calcification $(p=0.002)$ and between mild calcification and severe calcification $(p=0.063)$.

The serum phosphate levels of no calcification group of patients were $5.419 \pm 0.626 \mathrm{mg}$ / dl, level of $5.290 \pm 0.656 \mathrm{mg} / \mathrm{dl}$ in mild calficiation group, and level of $10.047 \pm 0.774 \mathrm{mg} / \mathrm{dl}$ in severe calcification 
group. Based on the statistical test it was concluded that there was no difference in the average phosphate level to the degree of calcification $(p=0.723)$.

\section{Correlation Between Calcium Serum Level and Phosphate Level}

From this analysis it was found that serum calcium and serum phosphate had a significant relationship $(p=0.001)$ with a fairly strong relationship strength $(r=0.324)($ Table 4$)$

\section{Multivariate Analysis}

Variables with bivariate test results with $\mathrm{p}$ values $<0.25$ were included in further multivariate analysis. The multivariate analysis test used was a multinomial logistic regression test using the Backward method. Analysis was conducted by determining the degree of no calcification as a comparison reference

The results in table 5 show that none of the variables had a statistically significant effect on the predictions of mild calcification compared with no calcification $(p=0.346)$. As for the prediction of severe calcification compared with no calcification, age had a significant effect $(p=0.001)$. The duration of hemodialysis, calcium level, age and Fetuin-A have correlation significantly $(p=0.005 ; 0.018 ; 0.001$ and 0.004 respectively).

$L n=-12,812+0,091$ (age) $+0,025($ duration of $H D)+0,825$ (calcium level) $-0,005$ (Fetuin-A level )

(Please see equation in Supplemental Files)

\section{Discussion}

In this study the majority of the study subjects were male (59.3\%), this result was consistent with the research report of Honkanen E et al. and IRR (Indonesian Renal Registry) data, The incidence of male calcification was greater than women with accompanying risk factors such as smoking history, diabetes, and coronary heart disease. ${ }^{1}$

In this study there are more severe degree of calcification found in patients with chronic renal failure (43.4\%) because the study population was patients with stage V CKD with dialysis. From Kimura's research, et al. stated that one of the factors in severe blood vessel calcification is affected by the severity of CKD and also the duration of dialysis experienced by a person.4 This is also in line with the theory described by Herrmann. ${ }^{2}$

Based on the results of a comparison of the mean age, there were significant mean differences in severe calcification compared to normal blood vessels, the mean age of patients having severe calcification was aged 59.47 years while those who did not experience calcification were 49 years old. Research by Turkmen, et al in Turkey get more or less the same results where the average patient who has severe 
calcification degree is 56 years. ${ }^{6}$ Krauss, et al. explained that increasing age, the degree of calcification is increasingly severe in patients with chronic kidney disease. ${ }^{7}$ Research conducted by Barreto et al on 101 routine hemodialysis patients, stated that there was a positive correlation between age and coronary artery calcification which was statistically significant with moderate correlation strength $(r=0.57 ; p=$ 0.001). 8-9 In line with the study Coll et al stated that there was a significant relationship between age and calcification in regular HD patients $(p=0.001) .{ }^{11}$

This is because with increasing age, the risk of increased blood vessel calcification coupled with decreased kidney function. Including the role of the kidney as a calcification regulation, where maintaining extracellular calcium and phosphate concentration and its inhibitory factors. ${ }^{2}$ this is aggravated by chronic kidney disease where the process of calcification has occurred since the onset of chronic kidney disease. ${ }^{1}$

Based on the statistical test it was concluded that there was a difference in the average duration of HD to the degree of calcification. In the study, the duration of hemodialysis significantly affected the occurrence of calcification in the abdominal blood vessels. In accordance with the CORD Study (Calcification Outcome in Renal Disease) study conducted by Honkanen, et al. Showed that in 933 study samples with dialysis duration $\geq 38$ months found calcification (AAC (Abdominal Aortic Calcification) score $\geq 1$ ) through lateral lumbal radiology occurred in $81 \%$ of patients; the severity increased significantly from L1 to $L 4(P<0,0001)$ and affected all segments in $51 \%$ of patients. And the annual increased risk of calcification of dialysis patients reaches $11 \%{ }^{10}$

Based on the statistical test, it was concluded that there was a relationship between the history of DM and the degree of calcification $(p=0.049)$. The oxidation process and oxidative stress of the tissue formed as a product of increased glucose levels are a risk factor for increasing the degree of calcification. This situation also occurs in the study of Joachim, et al. ${ }^{10,11}$ The results of this study are in line with the research conducted by Coll et al where there was a significant relationship between DM patients with the occurrence of vascular calcification $(p=0.007) .{ }^{9}$

In this study, it was found that serum calcium had a significant relationship with the occurrence of vascular calcification, in severe and without calcification. Calcium plays a major role in regulating contraction and relaxation of vascular smooth muscle by maintaining extracellular calcium and phosphate concentration. ${ }^{13}$ In patients with chronic kidney disease, there is a relationship between impaired mineral metabolism (serum calcium and phosphorus levels), bone abnormalities (renal osteodystrophy) and vascular calcification. ${ }^{1}$ Disorders of mineral and bone metabolism are the underlying factors in the progression of vascular calcification in patients with chronic kidney disease. ${ }^{1}$

Based on the statistical test it was concluded that there was no difference in mean phosphate levels to the degree of calcification $(p=0.723)$. The results of this study are in line with Coll et al's research $(p=$ 0.66). ${ }^{9}$ although different from the research by Distanbanchong et al, which states that phosphorus affects calcification due to changes in calcium and phosphate balance in patients with chronic kidney 
disease, causing vascular calcification. ${ }^{14}$ This result occurs because the population of the study sample has been using phosphate binders. So that the phosphate level in the blood is not too high $(5.290+0.656$ in mild calcification, $5.412+0.774$ in severe calcification). As concluded by Chan, et al., Phosphate binders can reduce serum phosphorus levels, but do not change the risk of death caused by vascular disease, one of which is vascular calcification. ${ }^{15}$

There are not many studies that multivariately review the risk factors associated with the occurrence of calcification in addition to serum calcium, serum phosphate and Fetuin-A levels but in this study it was found that age, duration of hemodialysis, calcium levels and Fetuin-A were risk factors that significantly affected the occurrence of severe calcification compared with no calcification. $(p=0.001)$. None of the risk factors had a significant effect on the occurrence of mild calcification compared with no calcification $(p=0.180)$.

This study is in line with theory telling that Fetuin-A is a systemic inhibitor of ectopic or calcification dystrophy. The involvement of Fetuin-A in pathologic calcification has been confirmed in large amount of studies involving patients suffering from calcification dystrophy. ${ }^{2,16}$

\section{Advice on Tables}

Table 1: Subjects Characteristics 


\begin{tabular}{ll} 
Characteristic & $\mathrm{n}=113$ \\
\hline Sex, $\mathrm{n}(\%)$ & $67(59,3 \%)$ \\
Male & $46(40,7 \%)$ \\
Female & $57(22-78)$ \\
\hline Age (years), Median (Min-Max) & $47(27-229)$ \\
\hline Duration of HD, Median (Min-Max) & \\
\hline Diabetes History & $24(21,2 \%)$ \\
Yes & $89(78,8 \%)$ \\
\multicolumn{1}{c}{ No } & $9,8(8,0-12,0)$ \\
\hline Serum Calcium,mg/dl Median (Min-Max) & $5,392 \pm 0,636$ \\
\hline Serum phosphate, mg/dl Mean \pm S.D & $49(38,1 \%)$ \\
\hline Classification degree & $21(18,6 \%)$ \\
No calcification & $49,4)$ \\
Mild calcificatio & \\
\hline Severe calcification & \\
\hline
\end{tabular}

Table 2 Correlation between Study Subjects Characteristics and Blood Vessel Calcification Degree 
Characteristic

No Mild Severe

calcification

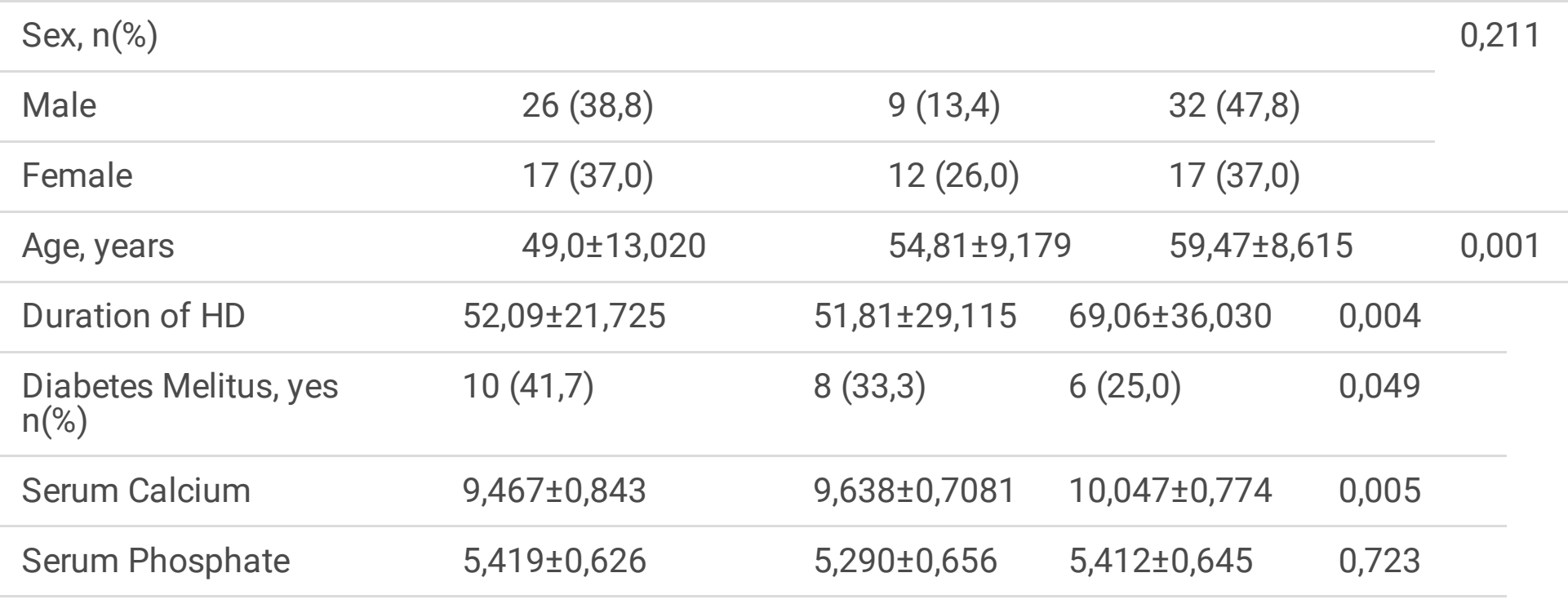

Table 3 Post Hoc Mann Whitney Test, Degree of Calcification in Research Subjects with Significant Difference of Means

\begin{tabular}{ll} 
Age & $\mathrm{P}^{\star}$ \\
\hline No calcification vs mild calcfication & 0,066 \\
\hline No calcification vs severe calcification & 0,001 \\
\hline Mild calcification vs severe calcification & 0,065 \\
\hline Duration of HD & \\
\hline No calcification vs mild calcification & 0,360 \\
\hline No calcification vs severe calcification & 0,007 \\
\hline Mild calcification vs severe calcification & 0,007 \\
\hline Serum calcium & \\
\hline No calcification vs mild calcification & 0,363 \\
\hline No calcification vs severe calcification & 0,002 \\
\hline Mild calcification vs severe calcification & 0,063
\end{tabular}


*Mann-Whitney Test

Table 4 Spearman Correlation Test for Calcium and Phosphate Level

\begin{tabular}{llll} 
& Variable & $r$ & $P$ \\
\hline Serum Calcium & Serum Phosphate & 0,324 & 0,001
\end{tabular}

Table 5. Multivariate Analysis of Multinominal Logistic Regression of Calcification Degree

\begin{tabular}{lllll} 
Variable & Coefisien & $p$ & OR & IK95\% \\
\hline Mild calcification, Intercept & $-3,461$ & 0,346 & & \\
\hline Severe calcification, Intercept & $-12,812$ & 0,001 & & \\
\hline Fetuin-A Levels & $-0,005$ & 0,004 & 0,995 & $0,991-0,998$ \\
\hline Duration of HD & 0,025 & 0,005 & 1,026 & $1,008-1,044$ \\
\hline Age, years & 0,091 & 0,001 & 1,096 & $1,039-1,155$ \\
\hline Calcium level & 0,825 & 0,018 & 2,281 & $1,151-4,522$ \\
\hline
\end{tabular}

Reference category : No calcification.

\section{Conclusions}

The conclusion of this study is that the duration of hemodialysis, diabetes mellitus, and calcium levels significantly influence the occurrence of calcification in the abdominal blood vessels. The prevalence of vascular calcification in hemodialysis patients who experienced severe calcification from this study was 49 people (43.4\%), there were no calcifications as many as 43 patients $(38.1 \%)$, and only 21 people who experienced mild calcification (18.6\%).

\section{Abbreviations}

CKD: Chronic Kidney Disease, HD: Hemodialysis, MSCT: Multi-Slice Computed Tomography, KDIGO: The Kidney Improving Global Outcome, DM: Diabetes Mellitus, IRR: Indonesian Renal Registry, AAC: Abdominal Aorta Calcification.

\section{Declarations}


This research was approved by Health Research Ethical Committee Medical Faculty of Universitas Sumatera Utara with Approval number 314/TGL/KEPK FK USU-RSUP HAM/2018. All patients that participate in this research were explained about the steps of the research. And it was written in a informed consent form that should be signed by participants.

\section{Conflict of Interest}

The authors report no conflicts of interest in this study.

\section{Funding}

This study was funded by all the authors them self.

\section{Authors Contributions}

All authors contributed toward data analysis, drafting and revising the paper, Riri A. Muzasti and Syafrizal Nasution gave a final approval of the version to be published and agree to be accountable for all aspects of the work.

\section{Acknowledgements}

The authors would like to thank to RSKG Rasyida, Medan (Rasyida Kidney Hospital, Medan) as a centre hospital to do this study.

\section{References}

[1]. Massy, Ziad A., and Tilman B. Drüeke. "Magnesium and outcomes in patients with chronic kidney disease: focus on vascular calcification, atherosclerosis and survival." Clinical kidney journal 5.Suppl_1, i52-i61. (2012): [2]. Herrmann, Marietta, and Wilhelm Jahnen-Dechent, "Pathology of ectopic calcification in fetuin-a deficient mice: compensatory gene regulation and mineralized matrix metabolism", Lehr-und Forschungsgebiet Zell-und Molekularbiologie an Grenzflächen, No. RWTH-CONV-125881, 2012. [3]. Jahnen-Dechent W, Heiss A, Schafer C, Ketteler M, "Fetuin-A regulation of calcified matrix metabolism", Circulation research, 108(12)1494-1509, 2011. [4]. NKF-KDIGO. “KDIGO clinical practice guideline for diagnosis, evaluation, prevention, and treatment of Chronic Kidney Disease-Mineral and Bone Disorder (CKD_MBD)". Kidney international Suplement, S1-130,2012 [5]. Kimura K, Saika Y, Otani H, Fujii R, Mune M, Yukawa S. "Factors associated with calcification of the abdominal aorta in hemodialysis patients", Kidney international Supplement, 71:S238-241, 2011 [6]. urkmen K, Gorgulu N, Uysal M, et al. "Fetuin-A, inflammation, and coronary artery calcification in hemodialysis patients", Indian Journal of Nephrology. 21 (2):90-94, 2011 [7]. Kraus MA, Kalra PA, Hunter J, Menoyo J, Stankus N,. "The prevalence of vascular calcification in patients with end-stage renal disease on hemodialysis: a cross-sectional observational study", Therapeutic advances in chronic disease, 6(3):84-96, 2015. [8]. Barreto DV, Barreto FC, Carvalho $A B$, et al. "Coronary calcification in hemodialysis patients: the contribution of traditional and uremiarelated risk factors", Kidney Int, 67(4):1576-1582, 2005 [9]. Coll B, Betriu A, Martinez-Alonso M, et al. 
"Large artery calcification on dialysis patients is located in the intima and related to atherosclerosis", Clin J Am Soc Nephro, 6(2):303-310, 2011 [10]. Honkanen E, Kauppila L, Wikstrom B, et al. "Abdominal aortic calcification in dialysis patients: results of the CORD study", Nephrology, dialysis, transplantation : official publication of the European Dialysis and Transplant Association - European Renal Association, 23(12):4009-4015, 2008 [11]. Ix JH, Katz R, de Boer IH, et al. "Fetuin-A is inversely associated with coronary artery calcification in community-living persons: the Multi-Ethnic Study of Atherosclerosis", Clinical chemistry. 58(5):887-895, 2012 [12]. x JH, Chertow GM, Shlipak MG, Brandenburg VM, Ketteler M, Whooley MA. "Association of fetuin-A with mitral annular calcification and aortic stenosis among persons with coronary heart disease: data from the Heart and Soul Study", Circulation, 15(19):2533-2539, 2007 [13]. Jahnen-Dechent W, Heiss A, Schafer C, Ketteler M. "Fetuin-A regulation of calcified matrix metabolism", Circulation research,108(12):1494-1509, 2011 [14]. Disthabanchong S. "Vascular calcification in chronic kidney disease: Pathogenesis and clinical implication", World journal of nephrology,1(2):43-53, 2012 [15]. Chan S, Au K, Francis RS, Mudge DW, Johnson DW, Pillans PI. "Phosphate binders in patients with chronic kidney disease", Australian prescriber, 40(1):10-14, 2017 [16]. Ketteler M, Bongartz P, Westenfeld R, et al. "Association of low fetuin-A (AHSG) concentrations in serum with cardiovascular mortality in patients on dialysis: a cross-sectional study. Lancet (London, England)", 361(9360):827-833, 2003

\section{Supplementary Files}

This is a list of supplementary files associated with this preprint. Click to download.

- equation.jpg 